\title{
Approche systémique du fonctionnement d'un territoire agricole bocager
}

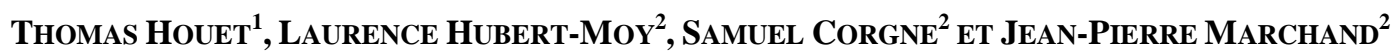 \\ thomas.houet@univ-tlse2.fr ; laurence.hubert@uhb.fr ; samuel.corgne@uhb.fr ; jean-pierre.marchand@uhb.fr \\ ${ }^{1}$ GEODE UMR CNRS 5602 - Université Toulouse 2, 5 allée Antonio Machado, 31058 Toulouse \\ ${ }^{2}$ COSTEL UMR CNRS 6554 LETG - Université Rennes 2, Pl. Rect. Henri Le Moal, 35043 Rennes
}

\section{RESUME}

Cet article présente une approche systémique du fonctionnement de territoires bocagers porteurs d'enjeux environnementaux. Après avoir analysé les dynamiques spatio-temporelles passées et actuelles des paysages, identifié et hiérarchisé les facteurs explicatifs des changements observés, l'analyse systémique met en évidence les échelles auxquelles ils se produisent et les différents acteurs qui interviennent dans l'évolution de ces paysages. Cette approche illustre la complexité des changements d'usage des sols dans des paysages très fragmentés en région agricole intensive et constitue un préalable à la simulation de changements futurs et à l'élaboration de politiques de gestion durable.

Mots-CLES : Usage des sols, Agriculture intensive, Composantes paysagères, Facteurs de changement

\section{AbSTRACT}

This article presents a systemic approach of the functioning of a landscape with a hedgerow network with environmental stakes. After having analyzed land-use/land-cover and landscape trends, identified and classified the driving forces of changes, the systemic analysis highlights the scales of changes and the stakeholders involved in the evolution of such landscapes. This approach shows the complexity of landuse change processes in intensive agricultural areas with a highly fragmented landscape. It constitutes an essential step to simulate future changes in agricultural practices and evaluate their environmental impacts. It's a key issue to implement sustainable policies.

KEY WORDS: Land use, Intensive agriculture, Landscape features, Driving forces

\section{Introduction}

Les changements intervenant dans les modes d'occupation et d'utilisation des sols engendrent des conséquences au niveau des processus qui contrôlent les écosystèmes. Par exemple, l'intensification de l'usage des terres, à travers l'emploi massif d'engrais et de produits phytosanitaires, entraîne des impacts environnementaux, parmi lesquels une forte dégradation de la qualité de l'eau et des sols. Dans certaines régions, les aménagements effectués sur le territoire agricole majorent et accélèrent les transferts de flux et de matières vers les cours d'eau, pouvant provoquer de graves phénomènes d'érosion des sols (Delahaye, 2002). Les effets de l'occupation et de l'utilisation des sols sur l'environnement dans des régions d'agriculture intensive, que ce soit dans des paysages ouverts de grandes cultures (O'Callaghan, 1996) ou dans des paysages de type bocager (Mérot et Jigorel, 1996) ont été démontrés. Ainsi, les changements qui s'y produisent, généralement subtils à l'échelle locale (arasement d'une haie, drainage d'une prairie humide...) et peu perceptibles à l'échelle de quelques décennies (Hubert- 
Moy, 2004) peuvent engendrer des conséquences environnementales non négligeables en terme d'érosion ou de dégradation de la qualité des eaux continentales (Deffontaines et al., 1997 ; Soulard, 2005).

Le contexte de la gestion de l'eau a considérablement évolué avec l'adoption en 2000 de la Directive Cadre Européenne sur l'eau (directive 2000/60/CE) qui impose, à l'horizon 2015, pour l'ensemble des Etats membres, « le bon état écologique des masses d'eau naturelles et le bon état potentiel pour les masses d'eau artificielles et profondément modifiées ». Elle se traduit dans la politique française de l'eau au sein des Schémas d'Aménagement et de Gestion des Eaux (SAGE). Pour élaborer des stratégies de gestion des ressources en eau qui soient efficaces et durables à l'échelle locale, les institutions qui instruisent les SAGE ont besoin de connaître les changements passés et actuels des modes d'usage des sols et des structures paysagères, d'identifier les facteurs qui les motivent, ainsi que les acteurs et les niveaux d'organisation impliqués.

L'analyse systémique, qui a pour intérêt d'appréhender un territoire comme un système dynamique, permettant de comprendre la dynamique des activités humaines dans un espace géographique, apparaît pour cela un outil privilégié (Le Berre, 1984). Ainsi, elle permet de modéliser conceptuellement le fonctionnement d'un territoire agricole en définissant les niveaux d'organisation auxquels se produisent les changements et par corollaire les acteurs avec lesquels la gestion de l'eau devra être pensée. Le fonctionnement du territoire est défini ici par les actions produites ou induites par l'ensemble des facteurs et des processus qui expliquent les changements du paysage ou d'une de ses composantes (occupation des sols, bocage, zone humide) durant une période donnée. L'originalité de la démarche proposée repose ici sur l'utilisation combinée de méthodes quantitatives et qualitatives, à dires d'experts et participatives dans le cadre d'une approche systémique globale.

En premier lieu, l'approche méthodologique utilisée pour déterminer le fonctionnement d'un système agricole bocager-type à partir de trois sites bocagers tests, sera présentée. En second lieu, nous exposerons les dynamiques spatio-temporelles reconstituées de ces trois sites d'études, les facteurs explicatifs de ces dynamiques, ainsi que la représentation du fonctionnement du système « territoire agricole bocager ».

\section{Approche méthodologique}

\subsection{L'approche systémique}

L'analyse systémique est une approche conceptuelle globale, formalisée ou non mathématiquement, décrivant plusieurs processus et leurs interactions. Les systèmes constituent ainsi un moyen de description et d'explication des interactions entre jeux d'acteurs et de contraintes. L'approche systémique appliquée en géographie cherche ainsi à établir l'ensemble des interactions spatio-temporelles entre des contraintes historiques, économiques, sociales, mentales, physiques, naturelles d'un territoire donné (Marchand, 1996). L'ensemble de ces interrelations agit sur le fonctionnement et la structuration de ce territoire, voire ceux d'autres territoires englobés ou englobants, voisins directs ou éloignés. Réciproquement, le territoire ainsi créé réagit à son tour sur les contraintes qui participent à son fonctionnement. 
La notion de «paysage » est appréhendée ici au sens de l'écologie du paysage (Burel et Baudry, 2003) mais à une échelle plus vaste. Le «paysage agricole bocager » fait référence au paysage visible, structuré par ses composantes ayant une ou plusieurs fonctionnalités (épuratrices, maintien de la biodiversité, ...) sur un territoire donné. Ces dernières peuvent, à travers les acteurs et les décideurs, influer sur le fonctionnement, la structure et l'évolution du territoire porteur. Ainsi, le «territoire agricole bocager »se construit à travers l'ensemble des actions qui participent à l'évolution d'un paysage agricole bocager, c'est-à-dire à travers l'anthroposystème agricole.

L'approche systémique doit fournir une clef de lecture indispensable à l'interprétation des changements observés. Ces modèles conceptuels sont indispensables au développement de modèles de simulation dynamique qui prennent en compte, dans leurs processus de modélisation, les différentes configurations spatiales de la surface du sol et les actions possibles que l'homme peut effectuer pour modifier l'utilisation du sol (Durand et al., 1983). L'approche systémique du fonctionnement d'un territoire agricole bocager repose sur deux étapes préalables : la détermination des trajectoires d'évolution de paysages bocagers et l'identification et la hiérarchisation des facteurs explicatifs des changements observés sur des sites d'études préalablement choisis.

\subsection{Les sites d'étude}

Les sites d'étude sont situés sur le bassin versant du Blavet, deuxième bassin versant de Bretagne par sa superficie $\left(2130 \mathrm{Km}^{2}\right)$. Un SAGE a été élaboré afin de faire face à des enjeux de gestion quantitative et qualitative de l'eau. Les trois sous-bassins versants retenus, d'une dizaine de $\mathrm{Km}^{2}$, sont représentatifs de la diversité du bassin versant du Blavet, tant du point de vue paysager, du paysage bocager dense au paysage remembré, que des systèmes de production agricole, de l'élevage laitier à tendance extensive à l'élevage intensif hors-sol (Figure 1).

La restauration de la qualité de l'eau représente un enjeu majeur pour l'ensemble des acteurs qui interviennent sur ces sites : ils sont situés dans des cantons en excédent structurel $^{1}$, qui sont des secteurs où les déjections des animaux représentent, une quantité d'azote supérieure à la capacité d'absorption des sols. Le site du Lestolet, situé dans la zone amont du Blavet, dans les Côtes d'Armor, est représentatif d'un paysage agricole au bocage dense et aux zones humides de fonds de vallées bien présentes. La plupart des exploitations agricoles sont spécialisées dans la production laitière, quelques unes possédant des ateliers hors-sol avicoles ou porcins. Le site du Coët-Dan situé dans la zone médiane du Blavet est représentatif d'une agriculture intégrée et intensive, basée sur le «modèle agricole breton » (Canévet, 1992). Les productions porcine et avicole y sont dominantes, souvent complétées avec une production laitière. Les paysages sont plus ouverts que sur le Lestolet, le bocage et les zones humides y étant aujourd'hui relictuels. L'agriculture sur le sous bassin versant du Stang Varric, est dominée par la production laitière et présente aussi quelques élevages hors-sol. Le paysage de ce site est caractérisé par une dichotomie marquée entre des plateaux ouverts et des versants et

\footnotetext{
${ }^{1}$ Un canton est considéré en excédent structurel dès lors que la quantité totale d'effluents d'élevage produite annuellement conduirait, si elle était épandue en totalité, à un apport annuel d'azote supérieur à $170 \mathrm{~kg}$ par hectare de surface épandable.
} 
des fonds de vallées largement boisées. L'hypothèse est faite que ces trois bassins versants sont suffisamment représentatifs de la diversité des territoires bocagers de la Bretagne pour que les conclusions puissent être étendues à d'autres espaces.
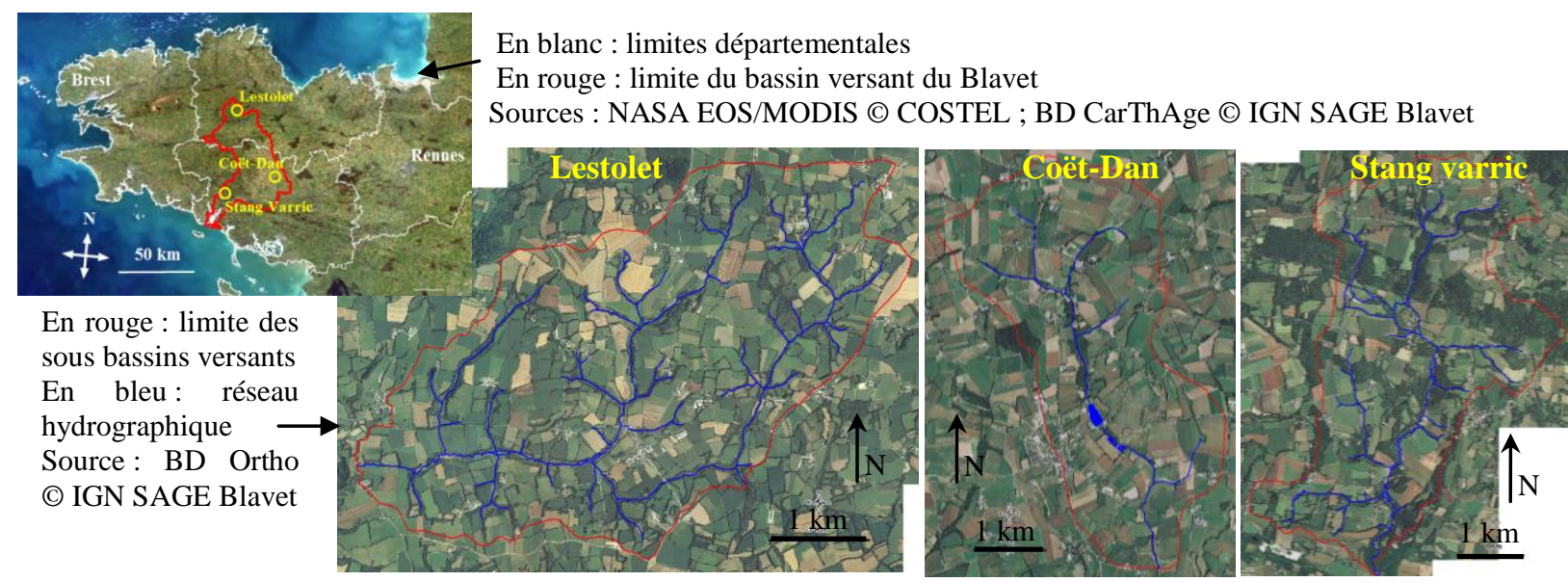

Figure 1. Localisation des sites d'étude

\subsection{Détermination des dynamiques paysagères}

La détermination des dynamiques paysagères été réalisée sur les trois sites d'études suivant deux dynamiques temporelles : un suivi décennal à partir de photographies aériennes sur les 50 dernières années (Temps Long) a permis de mettre en évidence les changements des usages des sols et des structures paysagères (Loveland et al., 2002); un suivi annuel à partir d'images satellitaires SPOT et IRS-LISS sur les 6-7 dernières années (Temps Court) a permis d'identifier les principaux systèmes culturaux (Tab. 1).

\begin{tabular}{|l|c|c|}
\hline 1. Descripteurs de l'évolution des structures paysagères & Temps Long & Temps Court \\
\hline Longueur du réseau linéaire boisé & $\mathrm{X}$ & \\
\hline Densité du réseau linéaire boisé & $\mathrm{X}$ & \\
\hline Densité du réseau linéaire boisé ayant un impact sur les transferts de flux de surface & $\mathrm{X}$ & \\
\hline Surface de la zone humide & $\mathrm{X}$ & \\
\hline Longueur de contact entre la zone humide et le versant drainé & $\mathrm{X}$ & \\
\hline Compacité des zones humides & $\mathrm{X}$ & \\
\hline Fragmentation des zones humides & $\mathrm{X}$ & \\
\hline 2. Descripteurs de l'évolution des modes d'usage des sols & Temps Long & Temps Court \\
\hline Taille du parcellaire & $\mathrm{X}$ & $\mathrm{X}$ \\
\hline Distribution de la taille du parcellaire & $\mathrm{X}$ & \\
\hline Ratio «SAU / non SAU » & $\mathrm{X}$ & \\
\hline Densité des surfaces bâties & $\mathrm{X}$ & \\
\hline$\%$ de surfaces boisées & $\mathrm{X}$ & \\
\hline \% de la SAU complantée (présence d'arbres -pommiers- dans les champs) & $\mathrm{X}$ & \\
\hline Evolution des principaux types d'occupation du sol pour les bassins versants & $\mathrm{X}$ & \\
\hline Evolution des principaux types d'occupation du sol dans les zones humides & $\mathrm{X}$ & \\
\hline Evolution des types d'occupation du sol à proximité des zones humides & $\mathrm{X}$ & \\
\hline Ratio « cultures / surfaces en herbe » & $\mathrm{X}$ & $\mathrm{X}$ \\
\hline$\%$ de la SAU en sols nus à peu couverts l'hiver & & $\mathrm{X}$ \\
\hline \% de sols nus suivis d'un maïs & & $\mathrm{X}$ \\
\hline Fréquence de sols nus à peu couverts en hiver sur la SAU & $\mathrm{X}$ \\
\hline Fréquence de sols nus en hiver suivis d'un maïs & $\mathrm{X}$ \\
\hline
\end{tabular}

Tableau 1- Descripteurs de l'évolution des structures paysagères et des modes d'usage des sols 
Une série de descripteurs statistiques et cartographiques destinés à déterminer l'évolution des modes d'occupation des sols et des structures paysagères influant le transfert de flux polluants a été dérivée des données de télédétection et d'un SIG (Tableau 1). L'analyse rétrospective effectuée à l'aide de ces descripteurs a permis de comparer les trajectoires d'utilisation des terres depuis un demi-siècle sur les trois sites.

\subsection{Identification et hiérarchisation des facteurs explicatifs des changements récents}

La seconde étape vise à déterminer les facteurs qui expliquent les changements paysagers observés et à évaluer leur poids respectif.

\subsubsection{L'identification des facteurs}

Une réflexion participative regroupant des acteurs locaux (agriculteurs, élus, gestionnaires) et des experts (agronomes, géographes, écologues, hydrologues), a permis de reconstruire les récits d'évolution de chacun des sites par grandes périodes clés et d'identifier une partie des facteurs expliquant les changements observés. L'inventaire des causes des changements a été complété ensuite par des entretiens individualisés avec des experts.

Les facteurs identifiés sont de nature très différente : ils peuvent être d'ordre qualitatif ou quantitatif, intervenir ponctuellement dans le temps ou agir sur plusieurs années ou décennies. La spatialisation des facteurs, nécessaire pour valider les facteurs identifiés à dire d'experts et désigner les variables influant réellement sur les évolutions constatées, est une étape délicate, car les données nécessaires à l'élaboration des descripteurs de ces facteurs sont très variées et pas toujours disponibles. Les facteurs identifiés ont une origine locale (parcelle agricole, exploitation agricole, commune, bassin versant), ou infra-régionale (bassin de vie, bassin de production), ou encore nationale ou internationale. Deux niveaux de représentation se dégagent : (1) un niveau local, compatible avec le niveau de précision des descripteurs des modes d'occupation des sols et des structures paysagères structurés au sein d'un SIG sur chacun des sites. Il s'agit essentiellement de descripteurs relevant du milieu physique ou des exploitations agricoles ; (2) un niveau communal, qui permet de replacer les changements observés sur les trois sites dans le cadre de l'évolution de l'ensemble du bassin versant du Blavet. En l'occurrence, aucun facteur explicatif de l'évolution des modes d'occupation des sols ne peut être traduit à l'échelle locale, car seules des données communales existent Recensements Généraux Agricoles de 1979, 1988 et 2000 (ONIC, 1979, 1988 et 2000).

Afin de valider les facteurs de changement des modes d'occupation des sols et des structures paysagères identifiés, deux méthodes sont utilisées. La première concerne les données structurées à l'échelle communale : elle consiste à calculer un coefficient de corrélation entre le descripteur du facteur explicatif et le descripteur de l'évolution des paysages (Tableau 1). La seconde concerne les données spatialisées à l'échelle locale : elle consiste à calculer une matrice de confusion $^{2}$ avec le SIG, en croisant les données

\footnotetext{
${ }^{2}$ La matrice de confusion, aussi connue sous les termes «matrice d'erreur» ou «tableau de contingence », est un outil statistique permettant de comparer deux cartes thématiques (une variable catégorielle $\boldsymbol{X}$ à prédire et une variable catégorielle $\boldsymbol{Y}$ considérée comme la variable de référence) en
} 
spatialisées du facteur explicatif avec les descripteurs paysagers.

\subsubsection{La hiérarchisation des facteurs}

Le poids respectif des facteurs influençant l'évolution des modes d'occupation des sols a été déterminé par deux approches : une qualitative et l'autre quantitative. La hiérarchisation qualitative des facteurs de changement a été effectuée par une analyse croisée des résultats issus des récits et des représentations spatialisées et validées de ces facteurs. Elle permet de dégager des principaux facteurs explicatifs des changements paysagers par site pour chaque période et par thème (occupation des sols, bocage, zones humides) et de définir leur influence (faible, moyenne, forte). La hiérarchisation quantitative des facteurs de changement n'est réalisable que lorsque le descripteur de l'évolution paysagère est à la même échelle spatio-temporelle que le descripteur des facteurs explicatifs des changements (Corgne, 2004-a et 2004-b). La détermination du poids respectif des facteurs influençant l'évolution des modes d'usages des sols a été effectuée à l'échelle communale, à travers une régression multiple standard appliquée pour chacune des dates d'observation (1979, 1988 et 2000). Enfin, les résultats ont été présentés lors d'une seconde réunion participative afin de les faire valider par les acteurs.

\section{Evolutions paysagères : trajectoires semblables, rythme et ampleur différenciés}

Les changements paysagers intervenus au cours des 50 dernières années sont succinctement analysés sur les trois sites d'étude à partir des descripteurs les plus représentatifs des évolutions observées.

\subsection{Les structures paysagères}

Le bocage a régressé depuis 50 ans d'environ $100 \mathrm{~m} / \mathrm{ha}$ entre sur les trois sites (Figure 2), mais de façon différenciée : le Lestolet a connu une baisse relativement continue du bocage, tandis que celui du Coët-Dan et du Stang Varric a très faiblement diminué jusqu'au début des années 1960, avant de reculer fortement avant les années 1980, pour ensuite se stabiliser.

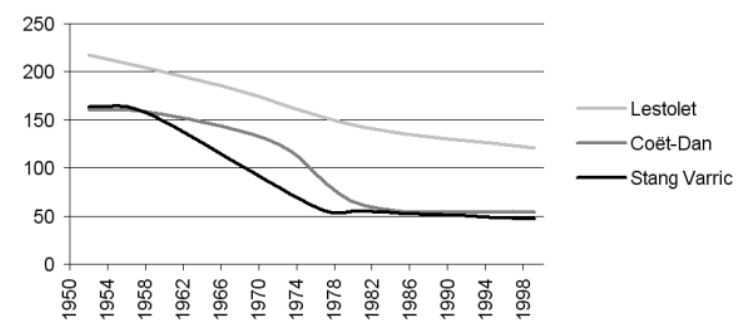

Figure 2. Evolution de la densité linéaire bocagère (m/ha) entre 1952 et 1999 sur les trois sites d'étude

mesurant leur degré de correspondance. L'objectif étant de produire un modèle permettant de prédire avec le plus de précision possible les valeurs prises par $\boldsymbol{X}$, les valeurs prédites sont confrontées avec les vraies valeurs prises par $\boldsymbol{Y}$, ce qui permet de déduire le taux d'erreur ou le taux de mauvais classement, qui est le rapport entre le nombre de mauvaises prédictions et la taille de l'échantillon. 
La place occupée par les zones humides de fonds de vallées en 1999 varie selon les sites : elle représentait 15,8\% (soit 219 ha) sur le Lestolet, mais seulement 7,9\% (soit 136,5 ha) et 5,3\% (soit 74,8 ha) sur le Stang Varric et le Coët-Dan où les opérations de drainage ont été plus importantes. Elles ont connu une régression spatiale continue sur les trois sites entre 1952 et 1999 (Figure 3), plus accentuée sur le Coët-Dan (-7,2\%) que sur le Lestolet $(-3,9 \%)$ et le Stang Varric $(-2,4 \%)$ ainsi qu'une fermeture prononcée de ces milieux.

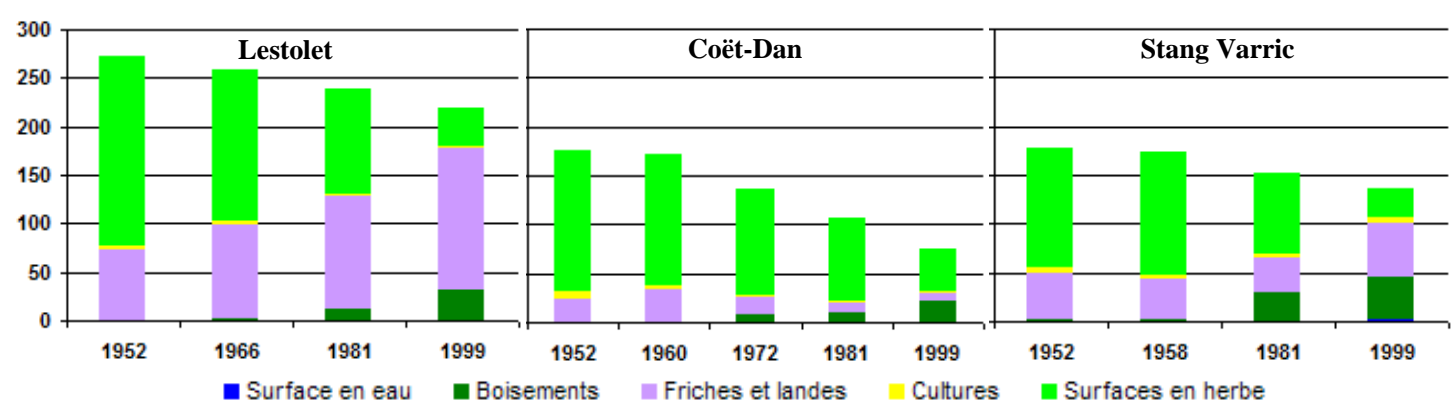

Figure 3. Evolution de la superficie (en ha) et des types d'occupation des sols des zones humides de fonds de vallées entre 1952 et 1999 pour les trois sites d'étude

Parallèlement à la régression du bocage et des zones humides, la taille des parcelles agricoles a sensiblement augmenté au cours de la période d'étude, mais suivant des rythmes différenciés selon les sites. La figure 4 montre l'agrandissement continuel de la taille du parcellaire agricole entre 1952 et 1999, qui a doublé pour le Lestolet, triplé pour le Coët-Dan et quadruplé pour le Stang Varric. Cet accroissement est plus marqué depuis les années 1970.

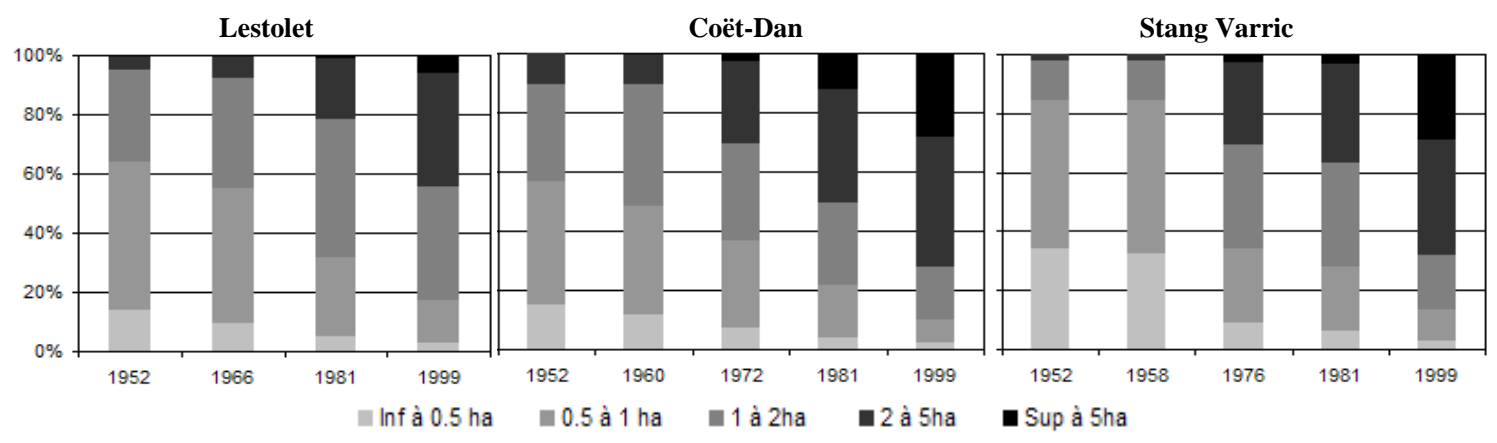

Figure 4. Evolution de la distribution de la SAU (en \%) par classes de taille de parcelle entre 1952 et 1999 pour les trois sites d'étude

\subsection{Les modes d'occupation des sols}

L'analyse des changements des modes d'occupation des sols réalisée parallèlement sur une période longue (1952-1999) et courte (1996-2002) montre que la surface agricole utile a légèrement diminué depuis 1952 sur les trois sites (Figure 5). Ceci tient d'une part à la fermeture des zones humides, où les friches et les bois ont augmenté au détriment des surfaces en herbe (Figure 3), mais également à la légère progression des 
surfaces bâties. Elle met également en évidence que la baisse des cultures par rapport aux surfaces en herbe s'amorce à la fin des années 1960 après une progression des cultures jusqu'à la fin des années 1950. Cette baisse, très marquée durant les années 1970, fait passer les proportions de 1 ha de prairies pour 4.4 ha de cultures sur le CoëtDan à 1ha de prairies pour 1,5 ha en 1975. L'équité entre cultures et prairies est atteinte sur le Lestolet et le Stang Varric dans les années 1970. A partir des années 1980, la tendance s'inverse et la part de cultures augmente légèrement et de façon plus marquée pour le bassin versant du Coët-Dan.

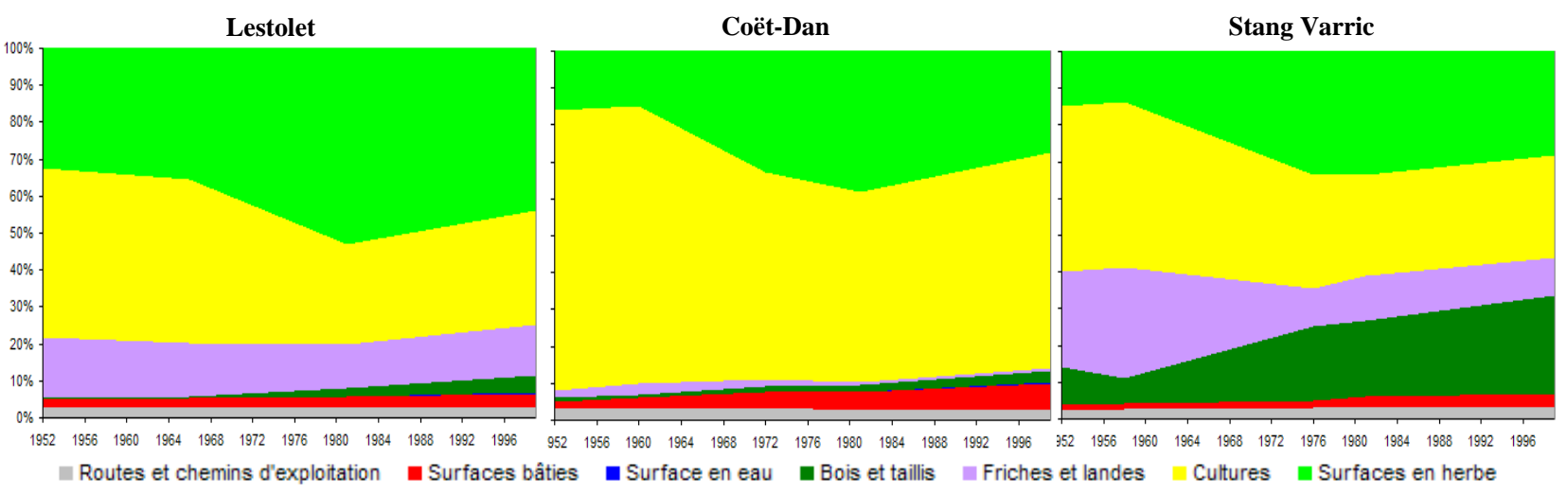

Figure 5. Evolution des principales classes d'occupation des sols (en \%) à l'échelle des trois sites d'étude 1952 et 1999

Enfin, l'évolution récente des pratiques agricoles vis-à-vis des transferts de flux, telle que l'implantation d'une interculture en hiver, s'effectue selon des trajectoires différenciées, l'évolution du Stang Varric et du Coët-Dan présentant cependant quelques similitudes (Figure 6).
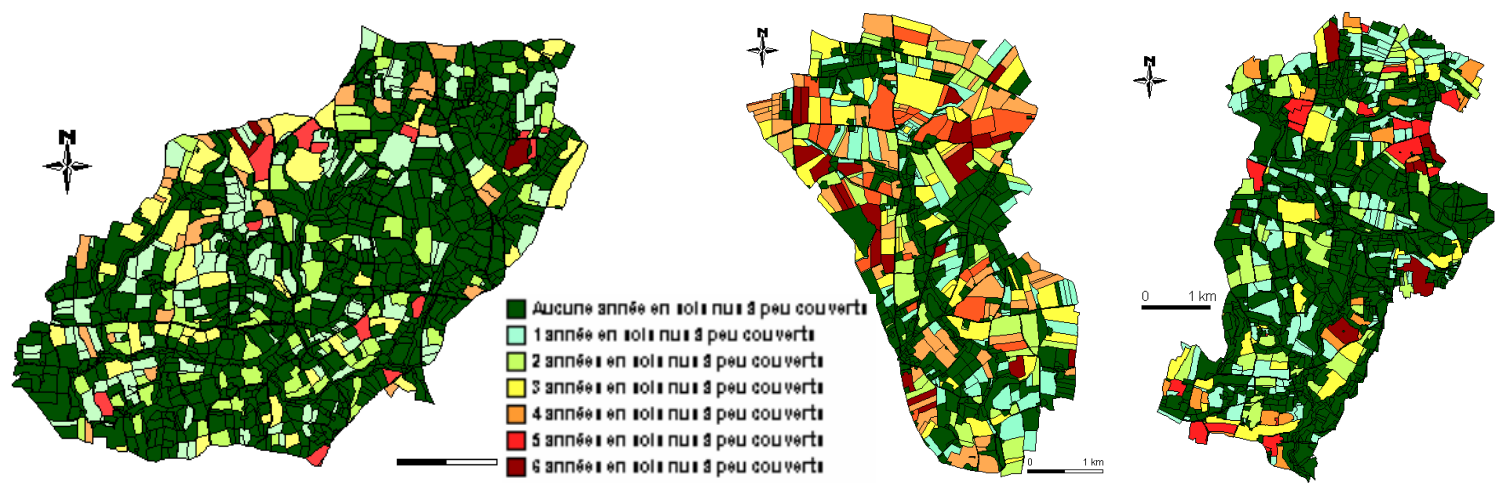

Figure 6. Cartographies de la fréquence de retour de la présence de sols nus à peu couverts depuis 1996 sur les trois sites d'étude

Ces sites ont donc connu globalement des évolutions semblables, tant au niveau de la transformation des structures paysagères que des changements des modes d'occupation des sols. Toutefois, l'ampleur et le rythme de ces changements diffèrent d'un site à l'autre, parfois très sensiblement. Ceci explique les différences observées actuellement, tant dans la part occupée par les différents éléments du paysage et par les cultures que 
dans leur répartition spatiale. L'ensemble de ces évolutions contrastées a généré des conditions différentes de transfert de flux d'eau et de polluants, certaines agissant comme un frein avec un rôle de tampon, d'autres comme un accélérateur favorisant les écoulements.

\section{Identification et hiérarchisation des facteurs explicatifs des changements récents}

Les résultats suivants présentent les facteurs des changements identifiés ainsi que leur implication sur l'évolution passée et actuelle (de 1982/83 à aujourd'hui) d'un territoire agricole bocager.

\subsection{Identification des facteurs : synthèse des récits d'évolution et validation}

Une réunion participative organisée le 14/10/2003 a permis d'identifier quatre grandes périodes d'évolution des territoires agricoles bocagers : (1) De 1945 à 1968/70 : D'un système semi autarcique aux débuts de l'intensification agricole ; (2) De 1968/70 à 1982/83 : La prospérité agricole avant les quotas laitiers ; (3) De 1982/83 à 1992 : Des quotas laitiers à la PAC de 1992 (4) De 1992 à aujourd'hui : L'adaptation de l'agriculture à la PAC de 1992 et l'émergence de préoccupations environnementales. Nous analyserons ci-dessous les deux dernières périodes: les changements amorcés depuis le début des années 1980 expliquent l'évolution actuelle des modes d'occupation et d'utilisation agricole des sols.

Les facteurs de changement identifiés ont été classés suivant sept grands domaines : le milieu physique, les progrès techniques, la démographie, le foncier, l'environnement, le domaine politique et réglementaire, la dimension culturelle et enfin la dimension économique. Ces facteurs ont été repris dans la figure 7, construite selon le modèle formel développé par Marchand (1986, 1996).

\subsubsection{La période 1982/83 - 1992}

La figure 7-a met en évidence les facteurs influant sur l'évolution des composantes paysagères sur les sites d'étude entre 1982/83 et 1992. La surproduction laitière, sousjacente depuis la fin des années 1970, a entraîné la mise en place des quotas laitiers entre 1982 et 1983. Cette réglementation a eu pour conséquence d'accentuer les spécialisations infra-régionales de production: les zones à dominante laitière se spécialisèrent vers une production de type « bovin lait-viande » avec ponctuellement des ateliers avicoles complémentaires; les zones à dominante hors-sol abandonnèrent leur production laitière pour s'intensifier, ce phénomène touchant a priori d'avantage les exploitations de petite taille et/ou ayant une production laitière faible. Cette spécialisation se produisit à l'échelle de l'exploitation agricole par l'adoption des OTEX (Orientation Technico-Economique des eXploitations). La baisse du nombre d'agriculteurs a donc engendré, après 1983, une intensification des productions sur les exploitations restantes.

L'orientation des exploitations vers un système de production de type «Bovin lait / viande » a entraîné une augmentation des surfaces fourragères pour s'adapter aux quotas laitiers, tandis que l'orientation des exploitations vers une production de type « Horssol » s'est accompagnée d'une augmentation nette des surfaces en cultures. Dans ce 
contexte, les zones humides situées dans les vallées étroites et encaissées se sont fermées en raison d'une absence de gestion, alors que les zones situées dans des vallées plus larges ont été soit drainées et utilisées comme pâtures, soit abandonnées, la baisse $\mathrm{du}$ nombre d'exploitants représentant une limite forte pour leur entretien. L'agrandissement des exploitations s'est effectué principalement par des échanges de parcelles ou par l'acquisition de surfaces libérées, les remembrements ayant été effectués en 1975-76 sur le Coët-Dan, et entre 1967 et 1972 sur le Stang Varric. Les actions individuelles de débocagement sont donc la cause principale de la baisse de la densité bocagère au cours de cette période, les haies occasionnant une gêne pour l'augmentation des productions agricoles.

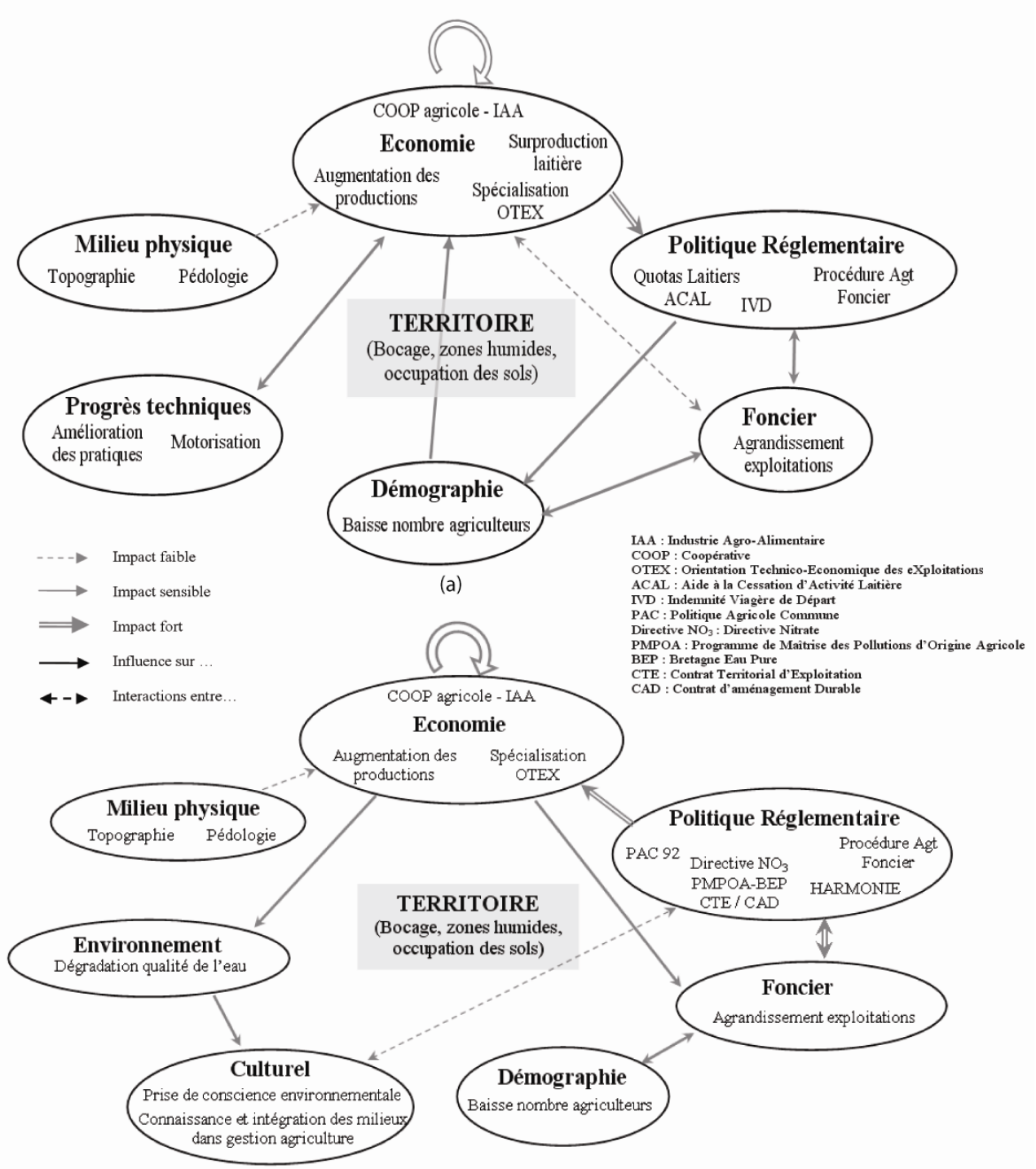

(b)

Figure 7. Facteurs et relations inter-facteurs influant sur l'évolution des structures paysagères et des modes d'occupations des sols d'un bassin versant entre (a) 1982/83 et 1992 et (b) 1992 et aujourd'hui. 


\subsubsection{La période 1992 à aujourd'hui}

La figure 7-b met en évidence les facteurs influant sur l'évolution des composantes paysagères sur les sites d'étude depuis 1992. La réforme de la PAC de 1992 a eu des effets significatifs sur l'évolution des modes d'occupations des sols. Le maïs fourrager a été encouragé dans les systèmes de type «Bovin lait viande » par le système de primes. Elle a aussi favorisé l'implantation de céréales ou de cultures légumières industrielles qui, associées au système «hors-sol», ont contribué à maintenir une certaine intensivité. Ces mesures ont accentué le drainage des zones humides jusqu'en 1994, là où le milieu physique le permettait. La baisse du nombre d'exploitants a favorisé l'abandon, l'enfrichement puis le boisement des fonds de vallées.

En parallèle, la multiplication des mesures réglementaires et des programmes de lutte contre la pollution des eaux d'origine agricole issus de l'émergence de facteurs culturels dans les années 1990, a influencé l'évolution de l'usage des sols: mise en place de plans d'épandage pour réduire les pollutions diffuses dans le cadre des PMPOA 1 et 2 ; conseils et incitations à l'amélioration de pratiques culturales (rotations culturales, couverture hivernale des sols...) dans les bassins versants BEP (Bretagne Eau Pure). L'évolution des modes d'occupation des sols se décline donc à l'échelle de l'exploitation agricole, souvent sur la base du volontariat (CESR de Bretagne, 2003).

Enfin, l'agrandissement des exploitations s'accompagne encore d'arasement de haies de façon ponctuelle et individuelle, mais les procédures d'aménagement foncier ont évolué et prennent désormais en compte les intérêts écologiques, anti-érosifs et paysagers du bocage. Des politiques de replantation de haies menées par la Région $\left(\right.$ Harmonie $^{3}$ ) et par le Conseil Général visant «la mise en valeur et l'amélioration du paysage rural et la préservation de l'environnement » sont appliquées depuis les années 1990 avec un léger effet à l'échelle communale et croissant au niveau intercommunal. Le programme $\mathrm{BEP}^{4}$ sensibilise aussi les exploitants à restaurer le bocage.

Le tableau 2 présente de façon synthétique, d'une part, l'ensemble des descripteurs des facteurs explicatifs des changements paysagers observés (leur dénomination, la source des données, leur échelle de représentation) et d'autre part, leur corrélation qualitative ou quantitative avec les descripteurs de l'évolution des paysages (tableau 1). Les résultats montrent que l'évolution des modes d'occupation des sols à l'échelle communale est fortement corrélée avec les types de productions bovine et porcine ainsi qu'avec le niveau d'intégration des exploitations agricoles à une économie productiviste (Marge Brute Standard par hectare - MBS/ha). A l'échelle locale, la répartition spatiale des types d'occupation des sols est corrélée à la taille des parcelles, les cultures étant implantées préférentiellement sur les parcelles les plus grandes, et dans une moindre mesure avec la topographie et les types de sols. L'évolution du bocage est fortement,

\footnotetext{
${ }^{3}$ Le programme régional Harmonie permet de financer des aménagements paysagers (plantation de haies). Le dernier programme Harmonie 4 a la volonté de mettre en cohérence « la fonctionnalité de l'agriculture avec l'amélioration et la préservation du milieu naturel ».

${ }^{4}$ Le programme Bretagne Eau Pure est un programme de reconquête de la qualité de l'eau opéré à l'origine sur quelques petits bassins versants stratégiques (alimentation en eau potable) et né de la volonté partagée de partenaires financiers institutionnels - l'Etat, le Conseil régional, les Conseils généraux bretons.
} 
mais pas exclusivement, corrélée à l'existence d'un remembrement, le Lestolet n'en ayant pas fait l'objet. Les haies qui n'ont pas été arasées sont principalement situées le long des routes et des bâtiments d'exploitations et contiguës à des prairies. L'évolution des zones humides est légèrement corrélée avec la baisse du nombre d'exploitants agricoles. Leur abandon est largement conditionné par leur accessibilité et le degré d'hydromorphie des sols.

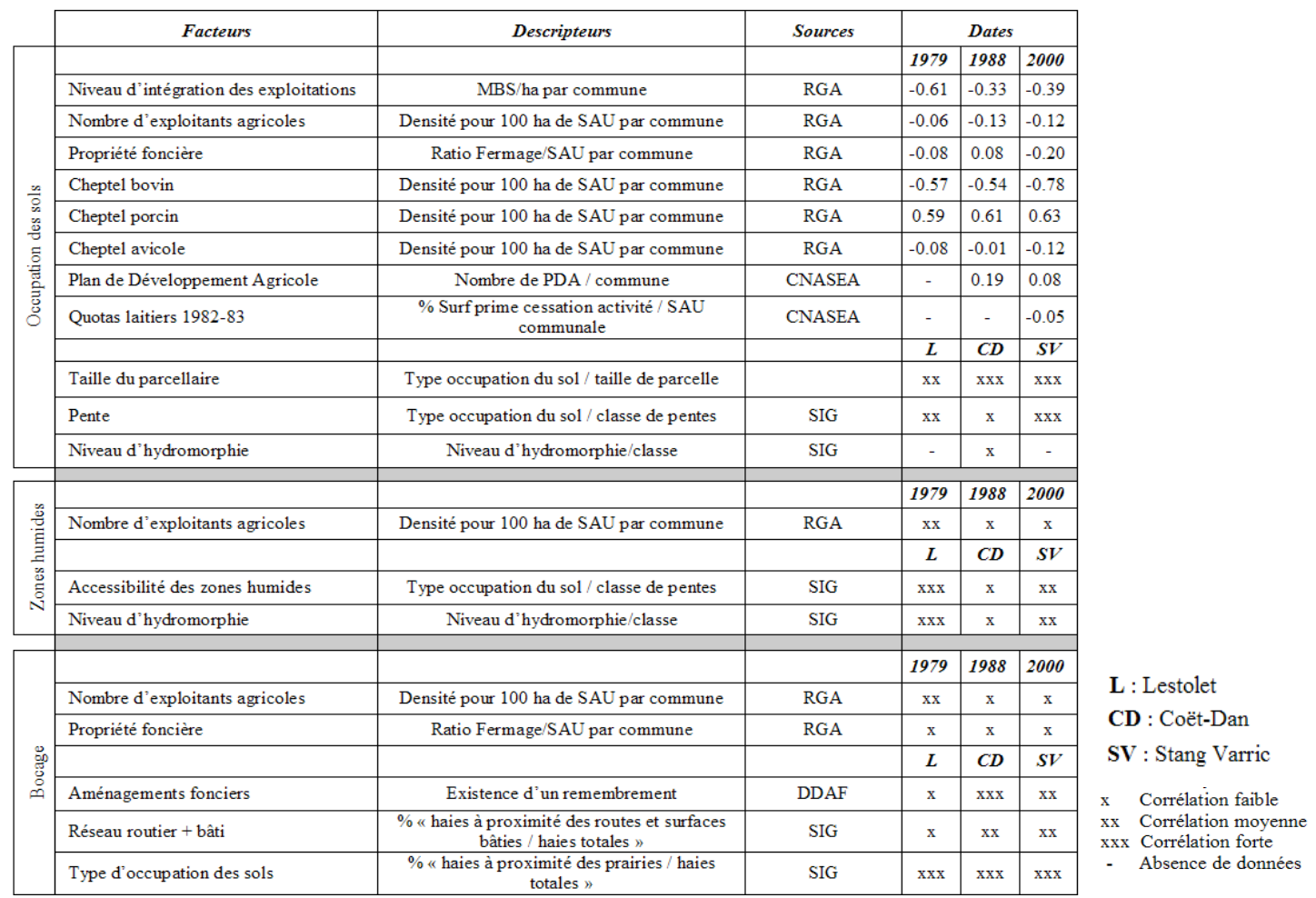

Tableau 2. Corrélation entre les descripteurs des facteurs explicatifs et les descripteurs des modes d'occupations des sols et des structures paysagères

\subsection{Hiérarchisation des facteurs : le rôle prépondérant du système de production}

Les résultats obtenus à partir de l'approche qualitative montrent que la mise en place des quotas laitiers apparaît comme le facteur principal de l'évolution des modes d'occupation des sols entre 1982/83 et 1992 (Tableau 3). Son influence a été variable selon les systèmes de production. La réduction des cheptels de vaches laitières a entraîné une baisse généralisée, plus ou moins forte, des surfaces en herbe. Cette baisse a été faible sur le Lestolet en raison de la diversification des exploitations vers la production de viande bovine, forte sur le Coët-Dan et le Stang Varric à travers respectivement la spécialisation dans la production hors-sol qui a engendré une augmentation des surfaces en maïs grain et la diversification des exploitations vers la production avicole. Le rôle du niveau d'intégration des exploitations agricoles a diminué, car l'ensemble des exploitations est désormais quasiment totalement intégré à la logique productiviste. La formation agricole ne possède plus de poids réel, car elle 
constitue aujourd'hui un préalable à toute installation. La baisse du nombre d'exploitants par le biais de l'IVD (Indemnité Viagère de Départ) ou de l'ACAL (Aide à la Cessation d'Activité Laitière) n'a pas d'influence directe sur l'usage des sols, mais indirecte de par son rôle sur l'agrandissement des exploitations.

\begin{tabular}{|c|c|c|c|c|c|c|c|c|c|c|c|c|c|c|c|}
\hline \multirow{3}{*}{ Facteurs } & \multicolumn{6}{|c|}{ Bocage } & \multirow{2}{*}{\multicolumn{3}{|c|}{$\begin{array}{c}\text { Zones humides } \\
1982 / 83 \text { à nos jours }\end{array}$}} & \multicolumn{6}{|c|}{ Occupation des sols } \\
\hline & \multicolumn{3}{|c|}{$1982 / 83$ à 1992} & \multicolumn{3}{|c|}{1992 à nos jours } & & & & \multicolumn{3}{|c|}{$1982 / 83$ à 1992} & \multicolumn{3}{|c|}{1992 à nos jours } \\
\hline & L & CD & Sv & $\mathrm{L}$ & CD & Sv & $\mathrm{L}$ & CD & Sv & L & CD & Sv & $\mathrm{L}$ & CD & Sv \\
\hline \multicolumn{16}{|l|}{ Economie } \\
\hline $\begin{array}{c}\text { Types de production } \\
\text { (OTEX) }\end{array}$ & & & & & & & $\mathbf{x}$ & $\mathbf{x}$ & $\mathbf{x}$ & $x$ & $\mathbf{x x x}$ & $\mathbf{x x}$ & $\mathbf{x}$ & $\mathbf{x x x}$ & $\mathbf{x x}$ \\
\hline $\begin{array}{l}\text { Niveau d'intégration } \\
\text { des exploitations }\end{array}$ & & & & & & & & & & $\mathbf{x}$ & $\mathbf{x x}$ & $\mathbf{x}$ & - & - & - \\
\hline \multicolumn{16}{|l|}{ Démographie/Culturel } \\
\hline Formation agricole & & & & & & & & & & - & - & - & - & - & - \\
\hline Perception ZH & & & & & & & $\mathbf{x}$ & $\bar{x}$ & $\mathrm{x}$ & & & & & & \\
\hline Perception bocage & $\mathbf{x}$ & $\mathbf{x x}$ & $\mathbf{x x}$ & $\mathrm{xx}$ & $\mathbf{x x}$ & $\mathbf{x x}$ & & & & & & & & & \\
\hline $\begin{array}{c}\text { Nombre agriculteurs } \\
\text { (baisse) }\end{array}$ & $\mathbf{x x}$ & $\mathbf{x x}$ & $\mathbf{x}$ & $\mathbf{x x}$ & $\mathbf{x x}$ & $\mathbf{x x}$ & $\mathbf{x}$ & $\mathbf{x}$ & $\mathbf{x}$ & $\mathbf{x}$ & $\mathbf{x x}$ & $\mathbf{x x}$ & $\mathbf{x}$ & $\mathbf{x x}$ & $\mathbf{x x}$ \\
\hline \multicolumn{16}{|l|}{ Foncier } \\
\hline Drainages & & & & & & & $x$ & $x x$ & $\mathrm{x}$ & & & & & . & \\
\hline Propriété foncière & $\mathbf{x}$ & $\mathbf{x}$ & $\mathbf{x}$ & $\mathbf{x}$ & $\mathbf{x}$ & $\mathbf{x}$ & & & & - & - & - & $\mathbf{x}$ & $\bar{x}$ & $\bar{x}$ \\
\hline \multicolumn{16}{|l|}{ Réglementaire } \\
\hline LOA $1960 / 62$ & & & & & & & & & & $\mathbf{x x}$ & $\mathbf{x x}$ & $\mathbf{x x}$ & - & - & - \\
\hline Quotas Laitiers & $\mathbf{x}$ & $\mathbf{x}$ & $\mathbf{x x}$ & $\mathbf{x}$ & $\mathbf{x}$ & $\mathbf{x x}$ & & & & $\mathrm{xxx}$ & $\mathbf{x x x}$ & $\mathbf{x x x}$ & $\mathbf{x x x}$ & $\mathbf{x x x}$ & $\mathbf{x x x}$ \\
\hline PDA & & & & & & & & & & $\mathrm{x}$ & $\mathbf{x x}$ & $\mathrm{xx}$ & $\mathbf{x}$ & $\mathbf{x x x}$ & $\mathbf{x x}$ \\
\hline Réforme PAC 92 & $\mathrm{xxx}$ & $x x x$ & $\mathrm{xxx}$ & $\mathrm{xxx}$ & $\mathrm{xxx}$ & $\mathbf{x x x}$ & & & & - & - & - & $\mathrm{xxx}$ & $\mathrm{xxx}$ & $\mathrm{xxx}$ \\
\hline CTE / CAD & $\mathbf{x}$ & & & & & & $\mathbf{x}$ & & & & & & & & \\
\hline Bretagne Eau Pure & $\mathbf{x}$ & & & & & & $\mathbf{x}$ & & & - & - & - & $\mathbf{x}$ & - & - \\
\hline \multicolumn{16}{|l|}{ Spatial } \\
\hline Localisation (versant) & $\mathbf{x}$ & $\mathbf{x x}$ & $\mathbf{x x}$ & $\mathbf{x}$ & $\mathbf{x x}$ & $\mathrm{xx}$ & & & & & & & & & \\
\hline $\begin{array}{c}\text { Proximité routes et } \\
\text { surfaces bâties }\end{array}$ & $\mathbf{x}$ & $\mathbf{x x}$ & $\mathbf{x x}$ & $\mathbf{x}$ & $\mathbf{x x}$ & $\mathrm{xx}$ & & & & & & & & & \\
\hline $\begin{array}{l}\text { Occupation du sol } \\
\text { (prairie adjacente) }\end{array}$ & $\mathbf{x x}$ & $\mathbf{x}$ & $\mathbf{x}$ & $\mathbf{x x}$ & $\mathbf{x}$ & $\mathbf{x}$ & & & & & & & & & \\
\hline $\begin{array}{l}\text { Milieu physique } \\
\text { (pentes / sols) }\end{array}$ & & & & & & & $x x$ & - & $x$ & $\mathbf{x}$ & - & $\mathbf{x x x}$ & $\mathbf{x}$ & - & $\mathbf{x x x}$ \\
\hline Distance au siège & & & & & & & & & & - & - & - & $\mathbf{x x}$ & $\mathbf{x x}$ & $\mathbf{x x}$ \\
\hline T aille du parcellaire & & & & & & & & & & $\mathbf{x}$ & $\mathbf{x x}$ & $\mathbf{x x}$ & $\mathbf{x x}$ & $\mathbf{x x x}$ & $\mathbf{x x x}$ \\
\hline
\end{tabular}

\section{L : Lestolet $\quad$ CD : Coët-Dan $\quad$ SV : Stang Varric}

Influence des facteurs : impact relatif entre les bassin versants sur une période Influence des facteurs : poids entre les facteurs pour une période - Influence nulle ou négligeable

$\mathrm{x}$ Influence faible

$\mathrm{xx}$ Influence moyenne

$\mathrm{xxx}$ Influence forte

Tableau 3- Hiérarchisation des facteurs explicatifs des changements récents des modes d'occupation des sols et des structures paysagères

Suite à la réforme de la PAC de 1992, la spécialisation des exploitations se poursuit et engendre des évolutions différenciées dans l'occupation des sols. Les primes de la PAC y contribuent fortement car elle concerne tous les systèmes de production : elle incite l'implantation du maïs fourrager pour les exploitations en système «bovin » et de céréales (blé) pour les exploitations hors-sol. L'évolution des modes d'occupation des sols dépend donc surtout de l'OTEX des exploitations. La localisation des types d'occupation des sols dépend: du milieu physique, notamment du niveau d'hydromorphie des sols et des pentes; de la taille du parcellaire, qui s'accroît à mesure que la structure parcellaire des exploitations s'agrandit; de la distance de la parcelle au siège d'exploitation, en lien avec l'agrandissement des exploitations, où les parcelles les plus éloignées du siège d'exploitation sont préférentiellement occupées par des cultures. L'évolution du bocage est surtout lié à l'agrandissement continuel des exploitations consécutif à la baisse du nombre d'exploitants : les arasements dus aux initiatives 
individuelles se multiplient, les remembrements de type «table rase " n'étant plus autorisés depuis les lois sur l'aménagement foncier et rural de 1985. La multiplication des mesures réglementaires visant le maintien, l'entretien et la replantation des haies tend à ralentir cette tendance. Les quotas laitiers de 1982/83 et la réforme de la PAC de 1992, du fait de leurs incitations à implanter des cultures et, dans une moindre mesure, l'agrandissement des exploitations, expliquent le prolongement de la régression du bocage : le débocagement concernant préférentiellement les haies situées au sein d'îlots de parcelles cultivées. Les Quotas Laitiers, et notamment la réduction du cheptel ou des surfaces de pâtures corrélée à la baisse de la démographie agricole, ont largement favorisé l'abandon des prairies humides et la fermeture de ces milieux. La PAC, incitant à l'implantation de cultures quel que soit le système de production, a entrainé des opérations de drainage des milieux humides. Son influence a été d'autant plus forte que certains drainages ont été subventionnés par les DDAF (Direction Départementale de l'Agriculture et des Forêts) jusqu'en 1994.

Les résultats obtenus à partir de l'approche quantitative (tableau 4) viennent confirmer les résultats issus de l'approche qualitative. Par exemple, les facteurs «cheptel bovin » et «cheptel porcin », représentatifs des systèmes de production, sont les plus corrélés et les plus significatifs avec l'évolution de l'occupation des sols, corroborant ainsi le poids prédominant des systèmes de production identifié par l'analyse qualitative.

\begin{tabular}{|c|c|c|c|c|c|}
\hline Année & $\begin{array}{l}\text { Coefficient de } \\
\text { corrélation }(R)\end{array}$ & Facteurs & Bêta & Signification & Commentaires \\
\hline \multirow{6}{*}{1979} & \multirow{6}{*}{0.873} & Niveau intégration & -0.502 & 0.000 & \multirow{6}{*}{$\begin{array}{l}\text { Les résultats trouvés pour } 1979 \text { témoignent du début d'une période de production } \\
\text { intensive. Des nuances se distinguent : la contribution significative du nombre } \\
\text { d'exploitants, qui par sa baisse, va plutôt dans le sens d'une augmentation des } \\
\text { surfaces en herbe (agrandissement etiou extensification); 1a contribution assez } \\
\text { significative du nombre de porcs et de volailles. La production "hors-sol " } \\
\text { porcine contribue à expliquer l'augmentation des proportions de cultures } \\
\text { (début de l'inversement de la tendance) sur certaines communes. La proprietté } \\
\text { foncière ne constitue pas une variable significative. }\end{array}$} \\
\hline & & Nbe Exploitants & -0.259 & 0.000 & \\
\hline & & Cheptel borin & -0.498 & 0.000 & \\
\hline & & Cheptel porcin & 0.202 & 0.012 & \\
\hline & & Cheptel avicole & -0.159 & 0.011 & \\
\hline & & Propriété foncière & 0.091 & 0.087 & \\
\hline \multirow{7}{*}{1988} & \multirow{7}{*}{0.784} & Niveau intégration & 0.237 & 0.100 & \multirow{7}{*}{$\begin{array}{l}\text { Les valeurs montrent clairement que le seul facteur explicatif prépondérant est } \\
\text { le système de production représenté par le nombre de porcs (Bêta }=0,699 \text { ) et le } \\
\text { nombre de bovins (Bêta }=-0,424 \text { ). Ces deux variables sont les seules } \\
\text { significatives. Les conséquences des quotas laitiers (réduction des cheptels et } \\
\text { baisse des surfaces en herbe) ont incité un nombre conséquent d'exploitants à } \\
\text { s'orienter vers un système hors-sol. Il est intéressant de noter que, bien qu'ayant } \\
\text { une fáible signification, le nombre de volaille influe désormais dans le sens d'une } \\
\text { augmentation des cultures. Le niveau d'intégration n'a plus de réelle } \\
\text { signification dans l'explication de l'occupation du sol : ces deux systèmes de } \\
\text { production peuvent engendrer un niveau d'intégration élevé alors qu'ils utilisent } \\
\text { les terres de façon opposée. }\end{array}$} \\
\hline & & Nbe Exploitants & -0.230 & 0.014 & \\
\hline & & Cheptel bovin & -0.424 & 0.000 & \\
\hline & & Cheptel porcin & 0.699 & 0.000 & \\
\hline & & Cheptel avicole & 0.020 & 0.843 & \\
\hline & & Proprieté foncière & 0.036 & 0.670 & \\
\hline & & PDA & -0.012 & 0.880 & \\
\hline \multirow{8}{*}{2000} & \multirow{8}{*}{0.884} & Niveau intégration & -0.103 & 0.431 & \multirow{8}{*}{$\begin{array}{l}\text { Là encore le système de production est le facteur explicatif dominant et } \\
\text { réellement significatif. Les quotas laitiers, ayant eu pour conséquence une } \\
\text { baisse continue du nombre de bovins, et l'impact de la PAC (implantation de } \\
\text { maiss fourrager) semblent avoir eu des répercussions deux fois plus fortes sur } \\
\text { l'evolution de l'occupation du sol pour des exploitations en systeme bovin (Bêta } \\
=-0,631 \text { ) que celles orientées dans un systeme «hors-sol » (Bêta }=0,330 \text { ). On } \\
\text { suppose que c'est la conjonction et de la baisse du nombre de bovins et de } \\
\text { l'augmentation des cultures qui expliquent ce résultat car les données de } \\
\text { télédetection ont montré une évolution plus forte des cultures sur le Coët-Dan } \\
\text { que sur le Lestolet. }\end{array}$} \\
\hline & & Nbe Exploitants & -0.161 & 0.013 & \\
\hline & & Cheptel borin & -0.631 & 0.000 & \\
\hline & & Cheptel porcin & 0.330 & 0.006 & \\
\hline & & Cheptel avicole & -0.112 & 0.165 & \\
\hline & & Propriété foncière & -0.022 & 0.433 & \\
\hline & & Plan développement & -0.015 & 0.713 & \\
\hline & & ACAL & -0.050 & 0.810 & \\
\hline
\end{tabular}

Tableau 4. Hiérarchisation des facteurs explicatifs des changements récents des modes d'occupation des sols et des structures paysagères

\section{Le système « territoire agricole bocager » : emboîtement d'échelles et d'acteurs}

La représentation du système «territoire agricole bocager» (figure 8) a mis en évidence (1) les échelles auxquelles les changements se produisent ainsi que les niveaux auxquels les décisions qui induisent de façon directe ou indirecte ces changements sont prises, et (2) les différents acteurs qui participent à l'évolution des paysages étudiés. 
Les facteurs explicatifs des changements relèvent de dimensions et d'échelles variées : une dimension géographique qui témoigne de l'influence de la configuration géographique d'un lieu; une dimension technique qui décrit les moyens mobilisables pour la gestion d'un territoire agricole; une dimension économique qui régule les productions à différentes échelles ; une dimension politico-réglementaire qui donne un cadre de conformité aux pratiques agricoles ou de gestion de l'eau; une dimension foncière qui fournit un cadre territorial à la gestion de l'espace ; une dimension humaine qui rend compte des dynamiques démographiques et socio-culturelles ; une dimension environnementale illustrant les enjeux de la gestion durable de ce type de territoire.

Le fonctionnement d'un système territorial tel qu'il est décrit par la figure 7 implique une structuration de l'espace en plusieurs niveaux d'organisations - ou «sphères » ayant leur propre fonctionnement. Ainsi, la figure 8 met en évidence l'emboîtement de trois sphères : une sphère micro-locale, celle de l'exploitation agricole (en jaune sur la figure) où se produisent les changements ; une sphère locale (en vert) où les entités de gestion de l'espace interviennent, enfin une sphère globale de régulation (en bleu) où sont adoptées des mesures réglementaires, politiques et économiques. Les facteurs -et leurs interactions- qui influent sur l'évolution des paysages sont décrits ci-après pour chacune de ces sphères organisationnelles. La détermination et la hiérarchisation des facteurs explicatifs des changements de ce système territorial et de son fonctionnement ont été validés par les acteurs lors de la seconde réunion participative (7/02/2005).

\subsection{La "sphère micro-locale»: l'exploitation agricole, niveau d'organisation clef des changements}

L'exploitant agricole, par ses activités de production, d'entretien et d'aménagement de son territoire, constitue le principal acteur des changements observés (Thenail, 1997); la mosaïque des exploitations explique l'évolution du paysage agricole. L'hydromorphie des sols, les pentes et la pédologie influent sur la localisation des modes d'usages des sols (Morlon, 2005 ; Mathieu et al., 2005). L'allocation spatiale des types d'occupation des sols, l'utilisation des zones humides de fond de vallée et l'entretien du bocage dépendent de la localisation des parcelles agricoles au sein de l'exploitation, et notamment de leur distance au siège d'exploitation (Morlon, 2005 ; Joannon et al., 2005). Les proportions des types d'occupation des sols et leurs modes d'allocation spatiale diffèrent d'un système de production à un autre. Les travaux de Baudry et al. (2003) et de Martin et al. (2006) ont montré que l'exploitant agricole est amené à adapter l'assolement et les règles d'allocation des cultures dès lors qu'ont lieu des modifications de la structure foncière et de la configuration spatiale d'une exploitation.

L'adoption d'un système de production dépend de plusieurs facteurs : du choix et des convictions de l'agriculteur; d'une demande économique en biens alimentaires; des potentialités agronomiques des terres et de la configuration spatiale de l'exploitation (éclatement ou regroupement des parcelles) ; des structures locales existantes (CUMA, ETA). Les enjeux en matière d'agrandissement foncier (augmentation des productions, gestion des épandages, etc.) fluctuent en fonction des systèmes de production des exploitations agricoles voisines en cessation d'activité. 


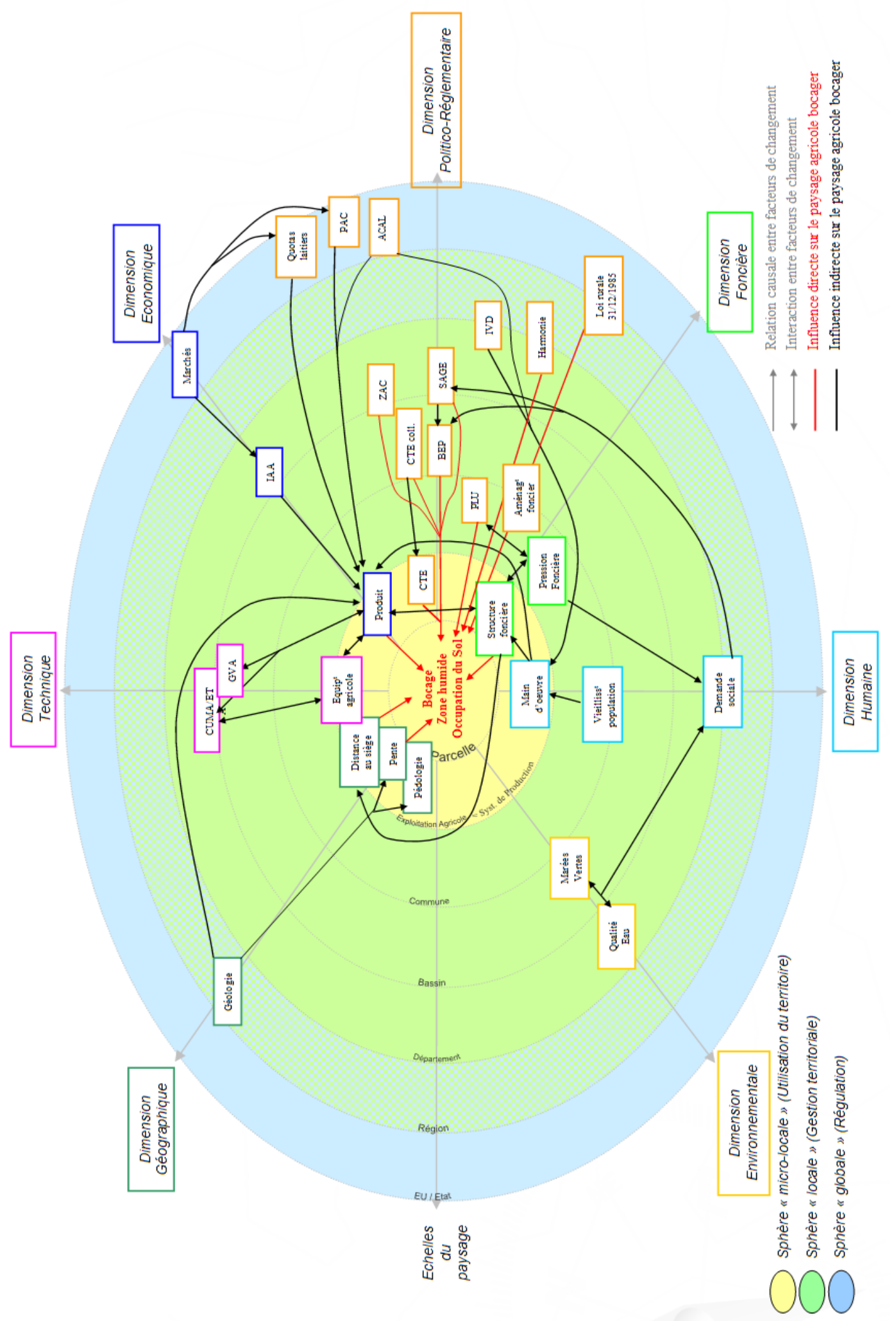

Figure 8. Représentation systémique du fonctionnement d'un territoire agricole bocager 


\subsection{La « sphère locale " : le rôle croissant des entités de gestion territoriale}

A ces échelles, ce sont les facteurs politiques et réglementaires et les entités de gestion territoriale qui sont prépondérants dans l'évolution d'un paysage agricole.

Les changements paysagers se produisent à la faveur d'actions enclenchées dans le cadre de programmes régionaux ou de directives européennes. Ainsi, à l'échelle du bassin versant, des mesures incitatives et d'accompagnement sont prises pour améliorer le rôle anti-érosif, hydrologique et épurateur du bocage, la préservation voire la restauration de certaines fonctionnalités des zones humides de fonds de vallées. Par exemple, à l'instar des CTE individuels, les CTE collectifs obligent les exploitants à gérer le bocage et les parcelles humides de leur exploitation. Les communes ou intercommunalités, dans le cadre d'aménagements fonciers ont un rôle direct sur les changements paysagers. Les PLU (Plan Local d'Urbanisme) influent aussi sur l'évolution de l'utilisation des terres en fonction de la détermination des parcelles constructibles et des zones naturelles telles que les parcelles humides de fonds de vallées. Des réglementations telles que les ZAC (Zones d'Actions Complémentaires) définies par la Directive Nitrates à l'échelle du canton, orientent les types d'occupation des sols en imposant à l'exploitant agricole d'implanter un couvert hivernal (une prairie, une culture d'automne, une Culture Intermédiaire Piège A Nitrates -CIPAN-, la repousse de la culture précédente, etc.).

\subsection{La "sphère -globale-de régulation": le poids prédominant de l'économie et des mesures politiques et réglementaires}

L'échelle de l'exploitation agricole, au travers des convictions et des choix de l'exploitant, est le niveau intégrateur de l'ensemble des politiques agricoles et environnementales et des textes réglementaires mis en place par l'Etat. Toute réforme de la PAC ou modification de la réglementation Européenne peut conduire à des évolutions de l'assolement, à des changements de système de production ou d'itinéraire technique (utilisation des parcelles humides...). Ainsi, la réforme de la PAC de 1992 a eu des conséquences importantes : le système de primes allouées à certaines cultures (céréales, maïs fourrager) a fortement encouragé leur implantation au détriment des prairies temporaires, dont la baisse a été limitée par la mise en place des Mesures Agri Environnementales (MAE) à la même date.

De même, les lois sur l'aménagement foncier et rural ont évolué et intègrent, depuis la loi du 31/12/1985, des mesures de protection des haies et talus renforçant les interdits en matière d'arrachage, de coupe d'arbres et de haies.

\section{Conclusion}

Cet article visait à définir le système « territoire agricole bocager ». La description et la compréhension de sa dynamique passée et de son fonctionnement actuel ont permis de mettre en évidence les niveaux d'organisation auxquels se produisent les changements et par corollaire les acteurs qui doivent être mobilisés pour une gestion durable de l'eau. Ainsi, les changements proviennent essentiellement de facteurs politiques et réglementaires qui se manifestent essentiellement à l'échelle de l'exploitation agricole. Les acteurs majeurs à l'échelle locale sont les agriculteurs, mais 
les gestionnaires de l'eau, les collectivités locales et les réseaux locaux (associations, ETA...) peuvent avoir un rôle non négligeable dans l'évolution d'un paysage agricole bocager.

L'originalité de ce travail repose sur la méthodologie employée pour représenter conceptuellement ce système territorial complexe. Elle fait appel à des approches diverses et complémentaires : télédétection, SIG, réunion participative, entretiens, statistique, analyse systémique. La détermination des trajectoires d'évolution des modes d'occupation des sols et des structures paysagères sur une cinquantaine d'années a été réalisée à l'aide d'un SIG et de données de télédétection. La présentation des résultats à des acteurs locaux lors d'une réunion participative a contribué à l'identification des facteurs explicatifs des changements observés. L'utilisation de données spatialisées et de l'approche systémique a permis de les hiérarchiser et de décrire le fonctionnement du système. A la fin de cette étape, un certain nombre de paramètres influant potentiellement sur les évolutions futures ont été définis et des questions-clés portant sur le devenir des territoires étudiés ont été déterminées. Cette démarche, appliquée à des territoires agricoles bocagers, peut tout à fait être employée sur des territoires présentant des paysages et des enjeux de gestion différents.

Cette approche permet d'appréhender la complexité des processus de changement d'usage des sols et des structures paysagères dans des régions agricoles intensives au paysage très fragmenté. Elle vient corroborer les recherches actuelles en agronomie portant sur l'analyse et la modélisation des relations entre territoires d'exploitations et paysage (Martin et al., 2006). Elle contribue également à l'élaboration de mesures d'actions et d'accompagnement visant à une meilleure gestion de la ressource en eau en identifiant des leviers d'actions (ex : CUMA, ETA) pouvant intervenir dans l'entretien des composantes paysagères. Enfin, elle constitue un préalable nécessaire à la simulation de changements de pratiques agricoles (Gaucherel et al., 2006) et corrobore des travaux antérieurs portant l'évaluation des impacts environnementaux de l'évolution des paysages agricoles (Thenail, 1997 ; Baudry and Thenail, 2004, Soulard, 2005). Elle permet par exemple d'évaluer les répercussions possibles de la réforme de la PAC de 2006 ou encore de l'agrandissement tendanciel des exploitations (Houet, 2006-b) qui peuvent avoir des conséquences plus ou moins sensibles sur les usages des sols et, par corollaire, sur les ressources en eau.

\section{Remerciements}

Les auteurs tiennent à remercier l'Institution du SAGE Blavet, la Chambre d'Agriculture des Côtes d'Armor, la Communauté de Communes de Plouay, l'INRA et le CEMAGREF de Rennes pour la mise à disposition des données, ainsi que le financements d'une partie de ces recherches par l'ANR (Agence Nationale de la Recherche) - ADD (Agriculture et Développement Durable) - COPT (Conception d'Observatoire des Pratiques Territorialisés).

\section{Bibliographie}

Baudry J., Burel F., Aviron S., Martin M., Ouin A., Pain G. and Thenail C. (2003). "Temporal variability of connectivity in agricultural landscape: do farming activities help?", Landscape ecology, vol. 18, $\mathrm{n}^{\circ} 3$, pp. 303-314. 
Baudry J. and Thenail C. (2004). Interaction between farming systems, riparian zones, and landscape patterns: a case study in western France, Landscape and urban planning, vol. 67, pp. 121-129

Deffontaines J.P., Brossier J., Benoît M., Chia E., Gras F., Lemery B., Roux M. (1997). Agriculture et qualité de l'eau : l'exemple de Vittel. Dossiers de l'Environnement de l'INRA $\mathrm{n}^{\circ} 14$.

Burel F. and Baudry J. (2003). Landscape ecology: concepts, methods and applications, Science Publishers, $362 \mathrm{p}$.

Canévet C. (1992). Le modèle agricole breton, Presses Universitaires de Rennes, 397 p.

CESR de Bretagne (2003). Le défi de la qualité de l'eau en Bretagne, Conseil Economique et Social Régional de Bretagne, 483 p. (hors annexes)

Corgne S. (2004-a). Modélisation prédictive de l'occupation des sols en contexte agricole intensif: Application à la couverture hivernale des sols en Bretagne, Thèse de Doctorat, Université de Rennes 2, 230 p. http://perso.enst-bretagne.fr/ mercierg/articles/these/corgnethese04Final.pdf

Corgne S. (2004-b). «Hiérarchisation des facteurs de changements de l'occupation hivernale des sols, Application au bassin versant du Yar (Bretagne) », Norois, ${ }^{\circ}{ }^{193,}$ pp. 17-29.

Delahaye D. (2002). Apport de l'analyse spatiale en géomorphologie. Modélisation et approche multiscalaire des risques, Habilitation à Diriger des Recherches, volume 1, Université de Rouen, 259 p.

Durand M.-G., Le Berre M., Chamussy H., Uvietta P. (1983). Expérience de modélisation dynamique en géographie pour des interventions en aménagement du territoire: le modèle AMORAL., Cheminements systémiques, Du modèle AMORAL à une réflexion théorique en Géographie, pp. 5-40.

Gaucherel C., Giboire N., Viaud V., Houet T., Baudry J. and Burel F. (2006). "A domain specific language for patchy landscape modelling: the Brittany agricultural mosaic as a case study", Ecological Modelling, vol. 194, n¹-3, pp. 233-243.

Houet T. (2006-a). Occupation des sols et gestion de l'eau: modélisation prospective en paysage agricole fragmenté (Application au SAGE du Blavet), Thèse de Doctorat, Université de Rennes 2 - Haute Bretagne, 370 p. http://www.uhb.fr/sc_sociales/Costel/Thèse_Houet.pdf

Houet T. (2006-b). "Modélisation prospective de l'occupation du sol en zone agricole intensive : Evaluation par simulations dynamiques de l'impact de l'évolution des exploitations agricoles dans la France de l'Ouest », Norois, n¹98, pp. 35-47.

Hubert-Moy L. (2004). Occupation du sol et télédétection : de l'inventaire à la modélisation prédictive, Habilitation à Diriger des Recherches, Université de Rennes 2 - Haute Bretagne, 327 p. (hors annexes) http://www.uhb.fr/sc_sociales/Costel/HDR_Hubert-Moy.pdf

Joannon A., Souchere V. et Tichit M. (2005). Analyse de la gestion spatialisée de l'exploitation agricole à partir de l'utilisation du parcellaire, in Agricultures et Territoires, Ed. Laurent C. et Thinon P., Hermès Science, Lavoisier, pp. 155-174.

Le Berre. M (1984). Pour une modélisation systémique de la différenciation spatiale in Geopoint 84 « Systèmes et localisations », Groupe Dupont Avignon, pp. 83-89.

Loveland T.R., Sohl T.L., Stehman S.V., Gallant A.L., Sayler K.L. and Napton D.E. (2002). "A strategy for estimating the rates of recent United States land-cover changes", Photogrammetric Engineering \& Remote Sensing, vol. 68, n¹0, pp. 1091-1099.

Marchand J.-P. (1996). Les contraintes naturelles et l'organisation de l'espace in Géopoint 96 «Espace et nature dans la géographie aujourd'hui ?», Groupe Dupont, Avignon, pp. 9-15. 
Martin P., Joannon A., Mignolet C., Souchere V. et Thenail C. (2006). Chapitre 10. Systèmes de culture et territoires: cas des questions environnementales in L'Agronomie aujourd'hui, Ed. T. Doré, M. Le bail, P. Martin, B. Ney and J. Roger-Estrade, Editions Quae, pp. 253-283.

Mathieu A., Thinon P. et Havet A. (2005). Les enquêtes en exploitation agricole sur les usages du territoire, in Agricultures et Territoires, Ed. Laurent C. et Thinon P., Hermès Science, Lavoisier, pp. 93-113.

Mérot P. et Jigorel A. (eds) (1996). Hydrologie dans les pays celtiques, Actes du ler colloque interceltique d'hydrologie et de gestion des eaux, 8-11 juillet, Rennes, France, Institut national de Recherches en Agronomie, $470 \mathrm{p}$.

Morlon P. (2005). La dimension spatiale des pratiques agricoles: une approche agronomique, in Agricultures et Territoires, Ed. Laurent C. et Thinon P., Hermès Science, pp. 175-190.

O'callaghan J.R. (1996). Land use - the interaction of economics, ecology and hydrology, Chapman \& Hall, London, 200 p.

ONIC (1979). Recensement Général Agricole, Données Statistiques, Cd-rom.

ONIC (1988). Recensement Général Agricole, Données Statistiques, Cd-rom.

ONIC (2000). Recensement Général Agricole, Données Statistiques, Cd-rom.

Soulard C. T. (2005). Les agriculteurs et la pollution des eaux. Proposition d'une géographie des pratiques. Nature, Sciences, Sociétés, 13, pp. 154-164.

Thenail C. (1997). «Le rôle des exploitations agricoles dans l'évolution des paysages : un facteur essentiel des dynamiques écologiques », Ecologia Mediterranea, vol. 23, pp. 71-90. 